\title{
Associação das Manifestações Bucais com Variáveis Sociodemográficas e Clínicas em Mulheres com Câncer de Mama
}

\section{Association of Oral Diseases With Sociodemographic and Clinical Variables in Women With Breast Cancer}

\author{
MARIAAPARECIDAAMARAL MUSSO 1 \\ MARCELA VIEIRA CALMON ${ }^{2}$ \\ LARISSA DELL'ANTÔNIO PEREIRA ${ }^{3}$ \\ CAMILA BRANDÃO-SOUZA 4 \\ MARIA HELENA COSTAAMORIM ${ }^{5}$ \\ ELIANA ZANDONADE ${ }^{6}$ \\ MARIA HELENA MONTEIRO DE BARROS MIOTTO
}

\section{RESUMO}

Objetivos: Avaliar prevalência de doenças bucais e sua associação com variáveis sociodemográficas e clínicas em mulheres com diagnóstico de câncer de mama e em tratamento. Material e Métodos: Trata-se de um estudo analítico, transversal, composto por 89 participantes. Foi realizado exame clínico - cárie, xerostomia, gengivite e afta; entrevista utilizando um roteiro semiestruturado para coleta de dados. Realizou-se análise descritiva dos dados. Os testes exato de Fisher e qui-quadrado foram utilizados para verificar possíveis associações, considerou-se nível de significância de 5\%. Resultados: Mulheres acima de 50 anos de idade representaram $25,8 \%$ da população estudada, ensino fundamental incompleto (36\%), raça/cor branca $(64 \%)$, condição socioeconômica C $(57,3 \%)$ e renda de até dois salários mínimos $(38,2 \%)$. Houve predomínio da necessidade de uso de prótese $(60,7 \%)$ devido ao grande número de dentes perdidos (média de 17,9). As pacientes com maior faixa etária apresentaram maior prevalência de xerostomia e maior número de dentes extraídos. Conclusão: Houve alterações bucais de associação aos tratamentos do câncer, como a necessidade de próteses dentárias, xerostomia, gengivites e aftas. Com o aumento da faixa etária, aumentouse também a extração dos dentes, a prevalência da xerostomia e da gengivite. Xerostomia esteve associada a raça/cor branca e uso de anti-hipertensivos.

\section{DESCRITORES}

Saúde Bucal.Cárie Dentária.Xerostomia.Gengivite.Neoplasias da Mama.

\begin{abstract}
Objective: To evaluate the prevalence of oral diseases and their association with sociodemographic and clinical variables in women diagnosed with breast cancer undergoing treatment. Material and Methods: This is an analytical, crosssectional study, composed of 89 participants. Clinical examination was performed to check for dental caries, xerostomia, gingivitis and cold sore. Participants were interviewed using a semistructured script and the data were analyzed descriptively. Fisher's exact and chi-square tests were used to verify possible associations, with a $5 \%$ significance level. Results: Women aged over 50 years accounted for $25.8 \%$ of the study population, with a predominance of incomplete elementary school (36\%), White race $(64 \%)$, socioeconomic condition "C class" $(57.3 \%)$ and income up to two minimum wages $(38.2 \%)$. There was a predominance of the use of dentures $(60.7 \%)$ due to the large number of missing teeth (mean of 17.9). Older patients had a higher prevalence of xerostomia and a higher number of extracted teeth. Conclusion: There were oral alterations associated with cancer treatment, such as the need for dentures, xerostomia, gingivitis and canker sores. As the age range increased, tooth extraction, xerostomia prevalence and gingivitis were also increased. Xerostomia was associated with white race and use of anti hyper tensives.
\end{abstract}

\section{DESCRIPTORS}

Oral Health.Dental Caries.Xerostomia.Gingivitis.Breast Neoplasms.

Odontóloga na Prefeitura Municipal de Vila Velha. Espírito Santo. Brasil.

2 Coordenadora do Curso de Odontologia da Faculdade Pítágoras de Linhares. Espírito Santo. Brasil.

Referência Técnica do Registro de Câncer da Secretaria de Estado da Saúde - SESA. Vitória. Espírito Santo. Brasil.

Doutoranda pela Escola Paulista de Enfermagem/ Universidade Federal de São Paulo - EPE/UNIFESP. São Paulo. São Paulo, Brasil.

5 Professora Titular do Departamento de Enfermagem da Universidade Federal do Espírito Santo - UFES. Vitória. Espírito Santo. Brasil.

Professora Titular do Departamento de Estatística da Universidade Federal do Espírito Santo - UFES. Vitória. Espírito Santo. Brasil.

Professora Associada II do Departamento de Odontologia da Universidade Federal do Espírito Santo - UFES. Vitória. Espírito Santo. Brasil. 
$\mathrm{S}$ aúde bucal é condição importante na saúde geral e bem-estar do indivíduo, e seu comprometimento gera transtornos, como dores e desconforto ${ }^{1}$.

Seguindo uma tendência mundial, nota-se, no Brasil, as transições demográfica e epidemiológica que produzem como cenário uma população com elevado número de indivíduos adultos e idosos ${ }^{2}$. Estas transformações nem sempre vêm acompanhadas de modificações no atendimento às necessidades de saúde desse grupo populacional. Juntamente com o enveIhecimento populacional, a transição epidemiológica, caracterizada pelo aumento da incidência e mortalidade de doenças crônico-degenerativas, como o câncer, resulta no aumento da demanda dessa população por serviços de saúde ${ }^{3}$.

Dentre os vários aspectos da saúde, a saúde bucal merece atenção especial pelo fato de que, historicamente, os serviços odontológicos não possuem como prioridade a atenção ao grupo de pacientes oncológicos, que, da mesma forma que a população adulta, possui alta prevalência de cárie, doenças periodontais, patologias da mucosa bucal e de necessidade de próteses ${ }^{4-8}$.No que diz respeito aos adultos e idosos, em geral a redução no ataque de cárie é menos significativa, tendo em conta o caráter cumulativo das sequelas da doença ${ }^{4,6}$.

O câncer, em geral, tem apresentado uma alta incidência no decorrer dos anos, sendo o câncer de mama o segundo mais frequente a nível global, com cerca de 1,7 milhões de casos, o mais incidente entre as mulheres ${ }^{9}$. No Brasil, as estimativas para esse tipo de câncer nos anos de 2016 e 2017 foi de 57.960 novos casos. No estado do Espírito Santo a incidência será de 1.010 casos, o que significa 53.85 casos a cada 100.000 mulheres, dado que aumenta de forma alarmante quando consideramos apenas sua capital, Vitória, com 77.86 casos por 100.000 mulheres, o que significa mais 140 casos novos ${ }^{10}$.

É comum, em pacientes oncológicos submetidos à terapia antineoplásica, como a quimioterapia, o desenvolvimento de complicações orais agudas ou tardias. Dentre elas encontram-se a mucosite, xerostomia, as infecções fúngicas, bacterianas e virais, as cáries de radiação entre outras. Portanto, é imprescindível que os pacientes oncológicos sejam acompanhados antes, durante e após a terapia antineoplásica a fim de que o cirurgião-dentista possa elaborar um plano de tratamento adequado às suas necessidades, de forma a prevenir ou controlar a ocorrência dessas complicações ${ }^{6,11,12}$.

O presente estudo teve como objetivo avaliar as doenças bucais e possíveis associações com as variáveis sociodemográficas - faixa etária, raça/cor, renda e Classe Socioeconômica (CSE); e as comorbidades hipertensão e diabetes, nas mulheres com câncer de mama em tratamento no Hospital Santa Rita de Cássia, em Vitória - ES.

\section{MATERIAS E MÉTODOS}

Trata-se de um estudo analítico, transversal, realizado no Hospital Santa Rita de Cássia/ Associação Feminina de Educação e Combate ao Câncer (HSRC/Afecc), em Vitória, ES, hospital filantrópico de referência para o tratamento de câncer em todo o Estado.

A população foi composta por todas as mulheres em fase de preparo cirúrgico para o câncer de mama no HSRC/Afecc e que atendiam aos critérios de inclusão, entre os meses de janeiro de 2012 a janeiro de 2013, totalizando 89 pacientes.

Considerou-se como critérios de inclusão: mulheres com diagnóstico histopatológico de câncer de mama; em tratamento no HSRC; na fase de preparo da $1^{\mathrm{a}}$ cirurgia do câncer de mama; que ainda não tinham realizado nenhum tipo de tratamento para o câncer; maiores de 18 anos.Excluiu-se mulheres com mais de um tumor primário. Não houve recusa de nenhum convite para a participação no estudo.

As variáveis dependentes estudadas foram: Índice CPO-D (Dente Cariado Perdido Obturado),gengivite, xerostomia e afta.E as independentes foram: idade, raça/cor, condição socioeconômica e comorbidades - hipertensão e diabetes.

Utilizou-se a técnica de entrevista com registro em formulário para a coleta das variáveis sociodemográficas: idade, raça/cor, renda, condição socioeconômica, escolaridade e comorbidades. Realizou-se o exame clínico para a coleta das variáveis clínicas: cárie dentária, xerostomia, afta e gengivite.

Caracterizou-se a condição socioeconômica de acordo com a posse de bens de consumo e escolaridade do chefe da família, em classe $A, B, C, D$ e $E$, segundo critério ABIPEME ${ }^{13}$.As informações sobre raça/cor, renda, escolaridade e comorbidades foram auto-declaradas e as quanto ao estadiamento do câncer obteve-se no prontuário das pacientes. 


\begin{tabular}{|c|c|c|}
\hline Característica & Número & Percentual \\
\hline \multicolumn{3}{|l|}{ Faixa etária } \\
\hline Até 49 anos & 28 & 31,5 \\
\hline $50-59$ anos & 23 & 25.8 \\
\hline $60-69$ anos & 21 & 23.6 \\
\hline 70 anos ou mais & 17 & 19,1 \\
\hline \multicolumn{3}{|l|}{ Estado civil } \\
\hline Solteira & 9 & 10,1 \\
\hline Casada/União estável & 53 & 59,6 \\
\hline Separada & 7 & 7.9 \\
\hline Viúva & 20 & 22.4 \\
\hline \multicolumn{3}{|l|}{ Escolaridade } \\
\hline Até 3 série ensino fundamental & 32 & 36,0 \\
\hline Da 4 série até 7 série ensino fundamental & 20 & 22,4 \\
\hline Ensino fundamental completo & 14 & 15,7 \\
\hline Ensino médio completo & 15 & 16.9 \\
\hline Ensino superior completo & 8 & 9,0 \\
\hline \multicolumn{3}{|l|}{ Raça/cor } \\
\hline Branca & 57 & 64,0 \\
\hline Neara & 7 & 7.9 \\
\hline Parda & 25 & 28,1 \\
\hline \multicolumn{3}{|l|}{ Local de residência } \\
\hline Zona urbana & 64 & 71,9 \\
\hline Zona rural & 25 & 28.1 \\
\hline \multicolumn{3}{|l|}{ Condição socioeconômica } \\
\hline Classe B & 17 & 19,2 \\
\hline Classe C & 51 & 57,3 \\
\hline Classe D & 19 & 21.3 \\
\hline Classe E & 2 & 2,2 \\
\hline \multicolumn{3}{|l|}{ Teve trabalho no último ano } \\
\hline Sim & 48 & 53,9 \\
\hline Não & 41 & 46.1 \\
\hline \multicolumn{3}{|l|}{ Qual trabalho exercia } \\
\hline Funcionária & 23 & 25,8 \\
\hline Dona de casa & 17 & 19,2 \\
\hline Autônoma & 26 & 29,2 \\
\hline Outro & 23 & 25.8 \\
\hline \multicolumn{3}{|l|}{ Renda familiar } \\
\hline Até 1 salário mínimo & 21 & 23,6 \\
\hline De 1 a 2 salários mínimos & 34 & 38,2 \\
\hline De 2 a 3 salários mínimos & 10 & 11.2 \\
\hline De 3 a 4 salários mínimos & 10 & 11,2 \\
\hline De 4 a 5 salários mínimos & 8 & 9,0 \\
\hline 6 salários mínimos ou mais & 6 & 6,8 \\
\hline Total & 89 & 100,0 \\
\hline
\end{tabular}


Para o registro de cárie dentária utilizouse a metodologia da Organização Mundial de Saúde $(\mathrm{OMS})^{14}$. Também anotou-se a presença de xerostomia, afta e gengivite, bem como suas classificações.

Para o diagnóstico da xerostomia mediuse a produção de saliva, sem estímulos, cuspida durante um minuto, em um recipiente plástico milimetrado ( 0 a $3 \mathrm{ml})$. Realizou-se esse teste após o intervalo de 1,5 a 2 horas caso a paciente tivesse fumado, ingerido líquidos ou alimentos, ou ainda se tivesse lavado a boca. Classificou-se a produção de saliva não estimulada $>0,2 \mathrm{ml} / \mathrm{min}$ em Grau 1 ; produção de saliva não estimulada entre 0,1 a 0,2 $\mathrm{ml} / \mathrm{min}$ em Grau 2; produção de saliva não estimulada $<0,1 \mathrm{ml} / \mathrm{min}$ em Grau $3^{15}$.

Realizou-se o diagnóstico da afta através do exame clínico da mucosa bucal, levando-se em consideração que a afta é uma úlcera que pode surgir em qualquer ponto da cavidade oral: língua, lábios, gengiva, garganta e úvula ${ }^{16}$.

Diagnosticou-se a gengivite pelo Índice Gengival de Löe-Silness ${ }^{17}$. Classificou-se como: Ausência de inflamação (Grau 0); Inflamação leve (Grau 1); Inflamação moderada, sangramento na exploração (Grau 2); Inflamação grave: sangramento espontâneo e ulceração (Grau 3).

Realizou-se uma análise descritiva dos dados, calculou-se o índice de Dentes Permanentes Cariados, Perdidos e Obturados (CPOD) através da média e percentual dos componentes. As associações entre as características sociodemográficas e o índice CPOD se deu pelo teste exato de Fisher. Adotou-se o nível de significância de $5 \%$. Utilizou-se o Statistical Package for the Social Sciences - SPSS (Chicago-EUA)versão 20 para esta análise.

O projeto foi aprovado pelo Comitê de Ética em Pesquisa da Universidade Federal do Espírito Santo sob o número 273/11, em 14 de dezembro de 2011 e autorizado pela direção do HSRC/Afecc.

\section{RESULTADOS}

Participaram do estudo as 89 mulheres com câncer de mama que atendiam aos critérios de inclusão.

Pode-se observar na Tabela 1 as caracte- rísticas sociodemográficas das pacientes, em sua maioria acima de 50 anos de idade, casadas ou em união estável, ensino fundamental completo, raça/cor branca, em sua grande maioria da zona urbana, pertencentes à Classe $\mathrm{C}$, autônomas $\mathrm{e}$ com renda familiar menor que dois salários mínimos.

$\mathrm{Na}$ Tabela 2 observar-se o estado geral da saúde bucal das pacientes. Verifica-se que $54 \%$ não procurou um dentista nos últimos 12 meses. As pacientes que buscaram o profissional, fizeram por motivo de rotina, seguido por urgência. A necessidade por utilização de próteses foi alta (60,7\%), 20,2\% apresentaram xerostomia, 10,1\% apresentaram gengivite e $6,7 \%$ aftas.

A Tabela 3 demonstra o estado geral de saúde da população estudada. O sedentarismo foi característica predominante, $73 \%$ das mulheres que não realizavam nenhum tipo de atividade física, $27 \%$ consumiam bebidas alcoólicas, 6,7\% fumavam; a hipertensão foi a comorbidade mais prevalente, seguida do diabetes; o estadiamento do tumor predominante foi o II.

$\mathrm{Na}$ Tabela 4 observa-se que as pacientes tinham mais dentes extraídos (média de 17,9) do que obturados e cariados, ao passo que elevava-se a faixa etária, concomitantemente também aumentava-se a média de dentes extraídos.

Quando analisada as possíveis associações da xerostomia com as variáveis independentes, os resultados mostraram diferenças estatisticamente significantes em relação à faixa etária $(p=0,021)$ e raça/cor $(p=0,047) . O$ que demonstra que as pacientes brancas $(\mathrm{OR}=3,452)$ quando comparadas as raças negra e parda, e as com 60 anos ou mais $(O R=3,460)$ tinham cerca de três vezes mais chance de ter xerostomia (Tabela $5)$.

Quando analisadas as associações com as comorbidades, verificou-se que as pacientes que utilizavam medicamentos para hipertensão tiveram uma chance maior $(\mathrm{OR}=11,136)$ de ter xerostomia que aquelas que não utilizavam. (Tabela 5).

O estudo demonstrou que a associação entre gengivite e faixa etária foi estatisticamente significante $(p=0,029)$, sendo que as pacientes de 60 anos ou mais tinham cinco vezes mais chance $(O R=5,255)$ de ter gengivite comparadas às demais. 


\begin{tabular}{|c|c|c|}
\hline $\begin{array}{l}\text { Característica } \\
\text { Procura de profissional últimos } 12 \text { meses }\end{array}$ & Número & Percentual \\
\hline$C D$ & 40 & 44,9 \\
\hline Dentista prático & 1 & 1,1 \\
\hline Não procurou & 48 & 54,0 \\
\hline \multicolumn{3}{|l|}{ Motivo da procura profissional } \\
\hline Uraência & 14 & 34,1 \\
\hline Rotina & 23 & 56.1 \\
\hline Prevencão & 4 & 9.8 \\
\hline \multicolumn{3}{|l|}{ Necessidade de utilização de prótese } \\
\hline Precisa & 54 & 60,7 \\
\hline Não precisa & 35 & 39.3 \\
\hline \multicolumn{3}{|l|}{ Xerostomia } \\
\hline Grau I & 4 & 4,4 \\
\hline Grau II & 11 & 12.4 \\
\hline Grau III & 3 & 3,4 \\
\hline Não & 71 & 79.8 \\
\hline \multicolumn{3}{|l|}{ Genqivite } \\
\hline Grau I & 7 & 7,9 \\
\hline Grau II & 1 & 1.1 \\
\hline Grau III & 1 & 1,1 \\
\hline Não & 80 & 89,9 \\
\hline \multicolumn{3}{|l|}{ Afta } \\
\hline Sim & 6 & 6.7 \\
\hline Não & 83 & 93,3 \\
\hline
\end{tabular}

\begin{tabular}{|c|c|c|}
\hline \multicolumn{3}{|c|}{$\begin{array}{l}\text { Tabela } 3 \text { - Informações gerais de saúde de mulheres } \\
\text { diagnosticadas com câncer de mama. Vitória/ES, 2012/2013 }\end{array}$} \\
\hline Característica & Número & Percentual \\
\hline \multicolumn{3}{|l|}{ Realiza atividade física } \\
\hline Não & 65 & 73,0 \\
\hline 1 ou 2 vezes semana & 10 & 11,2 \\
\hline 3 ou mais vezes semana & 14 & 15.8 \\
\hline \multicolumn{3}{|l|}{ Ingere bebida alcoólica } \\
\hline Nunca & 65 & 73,0 \\
\hline Socialmente & 15 & 16.9 \\
\hline Raramente & 7 & 7,9 \\
\hline Diariamente & 2 & 2.2 \\
\hline \multicolumn{3}{|l|}{ Fumante } \\
\hline Nunca fumou & 71 & 79.8 \\
\hline Ex-fumante & 12 & 13.5 \\
\hline Fumante & 6 & 6,7 \\
\hline \multicolumn{3}{|l|}{ Comorbidades } \\
\hline Hipertensão & 37 & 41,6 \\
\hline Diabetes & 12 & 13.5 \\
\hline Doença cardiovascular & 2 & 2.2 \\
\hline \multicolumn{3}{|l|}{ Estadiamento } \\
\hline 0 & 10 & 11,2 \\
\hline I & 16 & 18,0 \\
\hline II & 38 & 42.7 \\
\hline III & 13 & 14,6 \\
\hline IV & 1 & 1.1 \\
\hline Ianorado & 11 & 12,4 \\
\hline
\end{tabular}


Tabela 4 - Média dos componentes do índice CPOD segundo dados demográficos de mulheres diagnosticadas com câncer de mama. Vitória/ES, 2012/2013

\begin{tabular}{|c|c|c|c|c|c|}
\hline \multirow{2}{*}{ Variável } & \multicolumn{5}{|c|}{ CPOD } \\
\hline & Híqido & Cariado & Obturado & Extraído & Ausente \\
\hline \multicolumn{6}{|c|}{ Faixa etária } \\
\hline Até 49 & 15.6 & 0.4 & 5.6 & 9.2 & 1.3 \\
\hline $50-59$ & 8.9 & 0.2 & 4.2 & 18.0 & 0.7 \\
\hline $60-69$ & 5.7 & 0.1 & 3.7 & 21.8 & 0.8 \\
\hline 70 e mais & 4.1 & 0.1 & 0.3 & 27.2 & 0.4 \\
\hline \multicolumn{6}{|l|}{ Raca/cor } \\
\hline Branca & 8,3 & 0,2 & 4,0 & 18,5 & 1,0 \\
\hline Neqra & 11,0 & 0,6 & 3,9 & 16.4 & 0,1 \\
\hline Parda & 11,2 & 0.1 & 3,3 & 16,8 & 0,6 \\
\hline \multicolumn{6}{|l|}{ CSE } \\
\hline Classe B & 9,2 & 0,1 & 7,9 & 13,2 & 1,6 \\
\hline Classe C & 9,9 & 0,2 & 2,6 & 18,7 & 0,5 \\
\hline Classe D & 8,1 & 0,3 & 3,3 & 19,2 & 1,1 \\
\hline Classe E & 6.5 & 0,5 & 2,5 & 22,5 & 0,0 \\
\hline \multicolumn{6}{|l|}{ Renda } \\
\hline Até $1 \mathrm{SM}$ & 10,5 & 0,4 & 2,8 & 17,4 & 0,9 \\
\hline $1-2 S M$ & 9,3 & 0.1 & 2.1 & 20.1 & 0.3 \\
\hline $2-3 S M$ & 6.0 & 0.0 & 4.4 & 20.7 & 0.9 \\
\hline $3-4 \mathrm{SM}$ & 7.3 & 0.2 & 3.6 & 19.5 & 1.4 \\
\hline $4-5 \mathrm{SM}$ & 11.1 & 0.3 & 7.3 & 11.3 & 2.1 \\
\hline $5+$ SM & 11,8 & 0.0 & 11.3 & 7.8 & 1,0 \\
\hline Geral & 9,3 & 0,2 & 3,8 & 17,9 & 0,8 \\
\hline
\end{tabular}

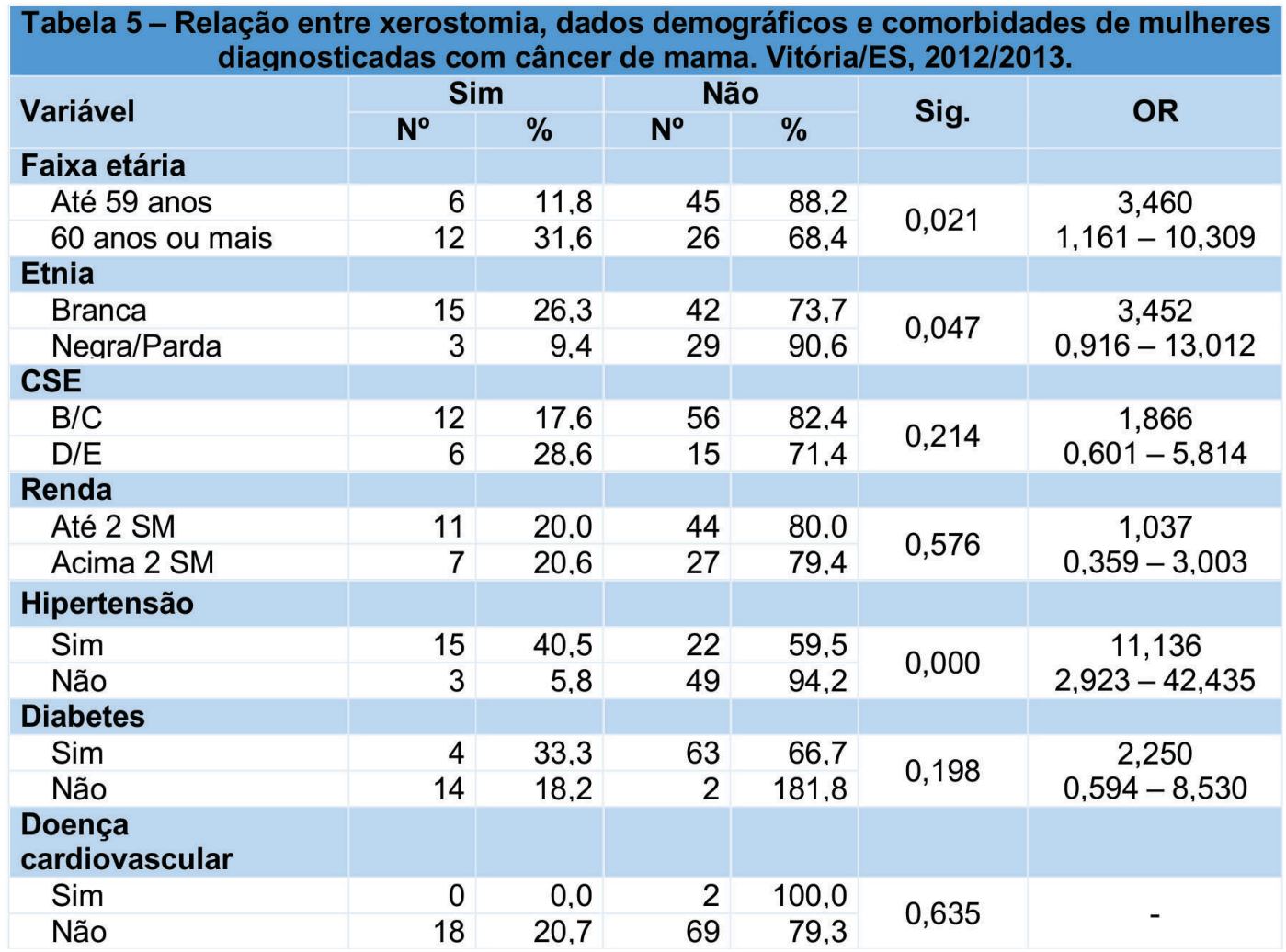




\section{DISCUSSÃO}

Os resultados do presente estudo confirmam achados anteriores que indicam que a saúde bucal dos brasileiros, em especial adultos e idosos, está muito longe de ser satisfatória ${ }^{18-20}$. Segundo as Diretrizes da Política Nacional de Saúde Bucal (DPNSB), é imprescindível o conhecimento do território e das pessoas nele inseridas, suas características sociais e econômicas, seus hábitos de vida (Tabelas 1 e 3), somente assim é possível entender como adoecem, e evitar suas enfermidades ${ }^{21}$.

A grande necessidade de próteses (Tabela 2), bem como a grande perda dentária na população de adultos e idosos (Tabela 4), são reflexo de problemas estruturais e estratégicos das políticas públicas de saúde. É fato que no Brasil, a limitação das ações de saúde bucal para adultos e idosos, grupos histori-camente pouco priorizados pelos modelos assistenciais, faz com que suas necessidades de tratamento se acumulem, acarretando perdas dentárias prematuras e grande demanda por tratamentos especializados, particularmente os protéticos ${ }^{16,18,22-25}$.

Políticas públicas com ênfase na atenção ao escolar são priorizadas, todavia não surtem os efeitos propagados: uma população adulta saudáve ${ }^{20}$. Este fato pode ser observado nos adultos e idosos de hoje, que apresentam elevado percentual de problemas periodontais e perda de elementos dentais, principalmente, a partir dos 40 anos de idade, o que é confirmado neste estudo cuja população tem em sua maioria 49 anos ou mais.Na DPNSB relata-se que os adultos muitas vezes apresentam dificuldade em frequentar o serviço de saúde bucal pelo horário de funcionamento, no qual muitas vezes estão trabalhando, e sugere $o$ atendimento em horários alternativos para essa população. No grupo dos idosos, a saúde bucal está diretamente ligada à qualidade de vida, deve-se facilitar o acesso ao serviço, diminuindo os trâmites burocrático, a exemplo, reservas de horários e dias específicos para atendimento dos mesmos ${ }^{21}$.

No Brasil, sabe-se que a odontologia curativa - por seu caráter privado, individualista e de altos custos - tornou-se inacessível a cerca de $90 \%$ da população brasileira ${ }^{19}$. O modelo assistencial odontológico público no Brasil, tem se caracterizado por uma atenção voltada para o tratamento cirúrgico-restauradordos sinais das doenças bucais mais prevalentes (cárie dentária e doença periodontal $)^{26}$, diferindo das demais ações de saúde pública, que focam na prevenção.

Existe no Brasil uma forte iniquidade em saúde bucal, com doenças se manifestando diferentemente de acordo com o grupo econômicosocial de inserção do indivíduo (Tabelas 4 e 5). A cárie e a doença periodontal, seguidas das lesões de mucosa, continuam causando dor e sofrimento para a população, sobretudo às classe socioeconômicas menos favorecidas, fenômeno agravado pela dificuldade de acesso aos serviços. $O$ acesso e a acessibilidade, quando existem, por vezes são restritivos, com prioridade da organização do serviço em solucionar demandas centradas nas queixas e doenças, enfatizando os procedimentos cirúrgicos restauradores ${ }^{20,23,27}$.

As associações entre as variáveis xerostomia e faixa etária, bem como xerostomia e uso de medicamentos hipertensivos obtiveram significância estatística (Tabela 5), corroborando com relatos de que medicamentos anti-hipertensivos, anti-histamínicos, diuréticos, tranquilizantes, reguladores de apetite, antipsicóticos, antiparkinsonianos, hipnóticos, anticolinérgicos, antieméticos e antidepressivos tem relação com a hipossalivação $0^{27,28}$.

A doença periodontal é a manifestação odontológica mais comum em pacientes diabéticos descompensados ${ }^{27}$. Este estudo não encontrou associação estatisticamente significante entre a gengivite e o diabetes, mas é sabido que doenças 
e alterações sistêmicas como diabetes, alterações cardiovasculares e pulmonares, distúrbios hormonais, infecções pelo HIV, entre outras podem agravar o quadro de gengivite já existente acelerando sua progressão e destruição tecidual, além de dificultar o tratamento ${ }^{29}$.

A associação entre o uso do tabaco e o acometimento pela gengivite também não obteve estatística significante, resultado que diverge de outros estudos realizados, onde relatam que o consumo do tabaco é um dos grandes responsáveis no desenvolvimento da gengivite apresentando não somente uma maior prevalência, mas também uma maior gravidade da doença em pessoas que fumam se comparado às não fumantes ${ }^{29}$.

A associação existente entre gengivite e 0 fator socioeconômico está diretamente ligado a renda e escolaridade e associam-se à sangramento gengival de maneira estatisticamente significante ${ }^{29}$. Indivíduos de menor renda e escolaridade comumentemente apresentam deficiência na escovação, sendo essa deficiência reconhecida como uma das principais causas de gengivite ${ }^{1,29}$. Neste estudo não foi possível provar essa relação, diferentemente da associação com a faixa etária, na qual comprovouse que idades mais elevadas tem associação com o acometimento por gengivite, e que mulheres acima de 60 anos têm pelo menos 5 vezes mais chances de serem acometidas por tal agravo.

Doença periodontal e condições inflamatórias crônicas são altamente prevalentes na população adulta ao redor do mundo, estimativas têm apontado para um aumento na ocorrência com o avançar da idade ${ }^{6}$. O atendimento odontológico e cuidados orais são importantes para eliminar fatores predisponentes às complicações provenientes durante e após o tratamento antineoplásico, como as infecções e os traumas. O processo da doença maligna em si e os efeitos colaterais do tratamento para o câncer comprometem a saúde oral e o estado nutricional do paciente $e^{6,12,29}$.

A partir dos efeitos adversos provocados pela radiote-rapia e quimioterapia, é importante ressaltar que a avaliação odontológica prévia a terapia antineoplásica é imprescindível, de maneira a observar o estado de saúde dental e periodontal do paciente, com consequente tratamento de alguma alteração existente, preparando a cavidade bucal para o início da tera-pia, prevenindo ou amenizando suas possíveis reações adversas ${ }^{11,12}$.

Medidas sociais e econômicas, voltadas ao enfrentamento da exclusão social e intervenções de saúde pública complementares, dirigidas aos grupos mais vulneráveis, tanto no campo da promoção da saúde bucal quanto no âmbito mais restrito da assistência odontológica, continuam a desafiar os formuladores e gestores de políticas públicas brasileiras ${ }^{30}$.

O cirurgião-dentista, juntamente à equipe multiprofissional de saúde, deve ter condições técnicas para elaborar estratégias de intervenção eficazes, prevenindo ou atenuando os sinais e sintomas oriundos dos tratamentos medicamentosos, contribuindo para o sucesso de todo o processo de recuperação da saúde e com a qualidade de vida destas pacientes.

Os estudos epidemiológicos de prevalência e severidade de doenças e condições bucais são importantes, pois devem subsidiar o planejamento de políticas preventivas e assistenciais de saúde bucal principalmente em grupos mais vulneráveis, como é o caso de pacientes oncológicos expostos à terapia antineoplásica. O que vem a desafiar os formuladores e gestores de políticas públicas brasileiras de saúde.

\section{CONCLUSÃO}

Conclui-se que houve alterações bucais de associação ao tratamento do câncer, como necessidade de utilização de próteses, xerostomia, gengivites e aftas. Os dentes extraídos foram um problema maior que os obturados e cariados, e aumentaram ao passo que a faixa etária também 
se elevou. Foi comprovada associação entre xerostomia e gengivite com faixa etária acima de
60 anos; e xerostomia com raça/cor branca e uso de anti-hipertensivos.

\section{REFERÊNCIAS}

1. Han K,Park J-B. Association between oral health behavior and periodontal disease among Korean adults: The Korea national health and nutrition examination survey. Medicine (Baltimore). 2017;96(7):e6176.

2. Fundo de População das Nações Unidas - UNFPA. Instituto Brasileiro de Geografia e Estatística - IBGE. Indicadores Sociodemográficos Prospectivos para o Brasil 1991-2030. Rio de Janeiro: Arbeit, 2006, p. 3639.

3. Silva JVF, Silva EC, Rodrigues APRA, Miyazawa AP. A Relação entre o Envelhecimento Populacional e as Doenças Crônicas Não-Transmissíveis: Sério Desafio de Saúde Pública. Enfermagem - Cadernos de Graduação - Ciências Biológicas e da Saúde. 2015; 2(3):91-100.

4. Brasil. Ministério da Saúde. Secretaria de Atenção à Saúde. Secretaria de Vigilância em Saúde. SB Brasil 2010: Pesquisa Nacional de Saúde Bucal: resultados principais / Ministério da Saúde. Secretaria de Atenção à Saúde. Secretaria de Vigilância em Saúde. - Brasília: Ministério da Saúde, 2012. 116p.

5. Mello ALSF, Erdmann AL, Caetano JC. Saúde bucal do idoso: por uma política inclusiva. Texto Contexto Enferm, Florianópolis 2008; 17(4): 696-704.

6. Seroli W, Rapoport A. Avaliação da saúde bucal no diagnóstico de pacientes com câncer bucal. RevBrasCir Cabeça Pescoço 2009; 38(3):157- 62.

7. Frazão P, Antunes JLF, Narvai PC. Perda dentária precoce em adultos de 35 a 44 anos de idade.RevBrasEpidemiol 2003; 6(1):49-57.

8. Moreira RS, Nico LS, Tomita NE, Ruiz T. A saúde bucal do idoso brasileiro: revisão sistemática sobre o quadro epidemiológico e acesso aos serviços de saúde bucal. Rev SaúdePública. 2005; 21(6):1665-75.

9. Ferlay, J. et al. Cancer incidence and mortality worldwide: Sources, methods and major patterns in GLOBOCAN 2012. Int J Cancer. Genève. 2015; 136(5):359-86.

10. Brasil. Ministério da Saúde. Estimativa 2016: Incidência de Câncer no Brasil / Instituto Nacional de Câncer José Alencar Gomes da Silva - Rio de Janeiro: INCA, 2015.

11. Paiva MDEB, Biase RCCG, Moraes JJC, Angelo AR, Honorato MCTM. Complicações orais decorrentes da terapia antineoplásica. Arq. Odontol. 2010; 46(1):4855.

12. Albuquerque RA, Morais VLL, Sobral APV. Avaliação clínica da frequência de complicações orais e sua relação com a qualidade de higiene bucal em pacientes pediátricos submetidos a tratamento antineoplásico. Arq. odontol.2007a; 43(2):9-16.
13. Brasil. Critério de Classificação econômica do Brasil/ 2008. ABEP, ABIPEME, 2007

14. Organização Mundial de Saúde. Levantamento Epidemiológico Básico de Saúde Bucal Manual de Instruções $4^{a}$ ed. Editora Santos, 1999, 53 p.

15. Feio M, Sapeta P. Xerostomia em cuidados paliativos. Acta Med. 2005; 18(1):459-66.

16. Neville BW, Damm DD, Allen CM, Bouquot JE. Patologia oral \&Maxilofacial. $2^{\circ}$ Ed. Rio de Janeiro:Guanabara Koogan,2004.Cap 9; 275-9.

17. Löe H, Silness J. Periodontal disease in pregnancy. I. Prevalence and severity. Acta Odont. Scand. 1963; 21(1):533-51.

18. Baldani MH, Almeida ES, Antunes JLF. Equidade e provisão de serviços públicos odontológicos no estado do Paraná. Rev Saúde Pública. 2009; 43(3):446-54.

19. Petry PC, Victora, CG, Santos IS. Adultos livres de cárie: estudo de casos e controles sobre o conhecimento atitudes e práticas preventivas, Cad Saúde Pública. 2000; 16(1): 145-53.

20. Palmier AC, Ferreira EF, Mattos F, Vasconcelos M. Saúde bucal: aspectos básicos e atenção ao adulto.NESCON/ UFMG.Curso de Especialização em Atenção Básica em Saúde da Família.Belo Horizonte:Coopmed, 2008.72p.

21. Ministério da Saúde. Secretaria de Atenção à Saúde. Departamento de Atenção Básica. Coordenação Nacional de Saúde Bucal. Diretrizes da Política Nacional de Saúde Bucal. Brasília, 2004. p. 03 e 14.

22. Matos DL, Giatti L, Costa MFL. Fatores sociodemográficos associados ao uso de serviços odontológicos entre idosos brasileiros: um estudo baseado na Pesquisa Nacional por Amostra de Domicílios. Cad Saúde Pública. 2004; 20(5):1290-7.

23. Shinkai RSA, Cury AADB. O papel da Odontologia na equipe interdisciplinar: contribuindo para a atenção integral ao idoso. Cad SaúdePública. 2000;16(4):1099109.

24. Holm-Pedersen P, Löe H. Textbook of Geriatric Dentistry. Copenhagen: Munksgaard.1996.

25. PuccaJúnior GA. Saúde bucal do idoso: Aspectos socais e preventivos. In: Gerontologia (M. Papaléo Neto org.), 1996, p. 297-310, São Paulo: Editora Atheneu.

26. Boing AF, Peres MA, Kovaleski DF, Zange SE, Antunes JLF. Estratificação sócioeconômica em estudos epidemiológicos de cárie dentária e doenças periodontais:características da produção na década de 90. Cad Saúde Pública. 2005; 21(3):673-78. 
27. Gallarreta FWM, Turssi CP, Palma-Dibb RG, Serra MC Histórico de saúde: atenção a condições sistêmicas e suas implicações, sobretudo nos fatores de risco de cárie. Rev OdontoCiênc.2008; 23(2):192-6.

28. Paunovich ED, Sadowsky JM, Carter P, 1997. The most frequently prescribed medications in the elderly and their impact on dental treatment. Dental Clinicsof North America. 1997, 41:699-726.

29. Seixas AR, Carvalho CO, Lima EB, Kowalczuk LL, Barckert ML, Silva TFO et al. Prevenção e Tratamento da Gengivite na Prática do Técnico em Saúde Bucal. Revista Gestão \& Saúde, 2010;1(2): 37-41.
30. Narvai PC, Frazão P, Roncalli AG, Antunes JLF. Cárie dentária no Brasil: declínio, polarização, iniqüidade e exclusão social. Rev Panam Salud Pública. 2006;19:385-93.

\section{CORRESPONDÊNCIA}

Nome: Maria Aparecida Amaral Musso

Endereço: Rua Vinícius Torres, 504, apt 504.

Praia da Costa, Vila Velha - ES.

CEP: 29101-105.

E-mail: aparecidaestrela2@gmail.com. 